\title{
MODELING INTERVENTION MEASURES AND SEVERITY-DEPENDENT PUBLIC RESPONSE DURING SEVERE ACUTE RESPIRATORY SYNDROME OUTBREAK*
}

\author{
SZE-BI HSU ${ }^{\dagger}$ AND YING-HEN HSIEH ${ }^{\ddagger}$
}

\begin{abstract}
The 2003 severe acute respiratory syndrome (SARS) epidemic came and left swiftly, resulting in more than 8,000 probable cases worldwide and 774 casualties. It is generally believed that quarantine of those individuals suspected of being infected was instrumental in quick containment of the outbreaks. In this work we propose a differential equation model that includes quarantine and other intervention measures implemented by the health authority, including those to prevent nosocomial infections and decrease frequency of contacts among the general public. We also consider the possible behavior change by the general populace to avoid infection, in response to the severity of the outbreak in general and to these intervention measures in particular. Complete analysis is given for the model without quarantine. For the general model with quarantine, a basic reproduction number is derived and full description of its dynamics is provided. We will show that introducing quarantine measures in the model could produce bistability in the system, thus changing the basic dynamics of the model. We give numerical examples of parameter values with which bistable steady states, where one is disease-free and the other endemic, could exist. However, realistic parameter values indicate that, assuming limited imported cases, the occurrence of the stable endemic steady state or bistability is unlikely. The modeling results indicate that for an infectious disease with infectivity and patterns of transmission typical of SARS, the outbreak can always be eradicated by implementing border control of imported cases and limited quarantine, along with the public's social response to avoid infections. Moreover, the results also suggest that quarantine measures will be effective in reducing infections only if the quarantined/isolated SARS patients and their potential contacts can successfully reduce their contact rate and/or transmission probabilities. Hence the effectiveness of quarantine for infectious diseases like SARS, for which no infection is being prevented during the quarantine period, can only be indirect and therefore must be combined with other intervention measures in order to quickly contain the outbreaks.
\end{abstract}

Key words. SARS, mathematical model, basic reproduction number, quarantine, bistable steady states, Taiwan

AMS subject classifications. 92D25, 92D30, 34D23, 93D20

DOI. $10.1137 / 040615547$

1. Introduction. The worldwide severe acute respiratory syndrome (SARS) epidemic outbreak of November 2002-July 2003 accounted for more than 8,000 infections with 774 fatalities directly attributable to SARS [1]. It is generally believed [2] that the experience of affected regions showed that the transmission of SARS-Coronavirus (SARS-CoV) can be effectively controlled by adherence to basic public health measures, including rapid case detection, case isolation, contact tracing, and good infection control such as hand-washing and use of personal protective equipment. Another measure believed to be instrumental in breaking the transmission chain is the quarantine of well but potentially infective individuals to prevent infections $[3,4,5,6]$. During the outbreak in Taiwan from April to June 2003, the

* Received by the editors September 22, 2004; accepted for publication (in revised form) June 7, 2005; published electronically January 6, 2006.

http://www.siam.org/journals/siap/66-2/61554.html

${ }^{\dagger}$ Department of Mathematics, National Tsing Hua University, Hsinchu, Taiwan (sbhsu@math. nthu.edu.tw). This author was supported by National Science Council of Taiwan grant NSC 932115-M-007-002.

${ }^{\ddagger}$ Corresponding author. Department of Applied Mathematics, National Chung Hsing University, Taichung, Taiwan (hsieh@amath.nchu.edu.tw). This author was supported by National Science Council of Taiwan grant NSC 93-2751-B005-001-Y. 
health authority attempted to quarantine more than 150,000 people who either had possible contact with a suspected SARS case or had just arrived from an affected area as determined by the World Health Organization (WHO). Of these quarantined individuals, only 17 were later officially confirmed as SARS cases. Hence questions remain as to the effectiveness of the quarantine.

During the outbreak, two distinct levels of quarantine were implemented in Taiwan. Level A quarantine, aimed at people suspected of having close contact with a suspected SARS case, was implemented on March 18, 2003. Level B quarantine, aimed at travelers from affected areas, was initiated on April 28 in the aftermath of the first SARS fatality in Taiwan on April 26. Details of the implementation of quarantine measures in Taiwan were described in [7]. By the end of the summer, a total of more than 150,000 people had been quarantined during the SARS outbreak.

There were 346 officially confirmed SARS cases as defined by WHO during the outbreak of 2003 in Taiwan, among which were 37 direct SARS casualties and 36 SARS-related deaths. In addition, 180 patients, who either had a previous negative PCR or antibody test or had been suspected or ruled-out cases, tested SARS antibody positive. However, Level B quarantine detected no confirmed SARS cases, while Level A quarantined persons included 17 officially confirmed SARS cases and 7 suspected or ruled-out cases with positive antibody tests [8]. Using the case data of the 480 laboratory-confirmed SARS cases, [8] showed that, compared to all other patients, previously quarantined persons had a significantly shorter onset-to-diagnosis time, i.e., the time it took a person with onset of symptoms to be diagnosed with suspected SARS and hospitalized. Hence quarantine had at least been useful in attaining more rapid detection and hospitalization of cases.

Rapid case definition also depends on knowledge regarding the clinical and molecular aspect of the disease, an inherently difficult task when facing a newly emerging disease like SARS. Contact tracing and quarantine of the traced contacts is another effective but difficult measure especially in an established democratic society, due to the ethical and legal ramifications [9]. Adherence to infection control, in the hospital or in the community, by the health care workers or the general populace, depends very much on the individual. The personal decision whether to diligently avoid contacts and infection is often based on the circumstances, i.e., whether there is any perceived cause for behavior change by the individual. The increasing severity of an outbreak or the implementation of massive intervention measures, e.g., the images of everyone wearing a face mask while in public places, is surely a cause for behavior change to avoid infection. This perhaps critically important factor will also be considered in our model.

In this work we will focus on three types of interventions evident during the past SARS outbreak: quarantine of potential infectives, isolation of suspected cases, and behavior change of the general public (including health care workers) in response to the increasing severity of the outbreak in an effort to avoid contacts which might lead to SARS infection. The focus is to study the roles played by intervention measures and social response in the quick containment of the outbreak. Previous modeling work of the SARS epidemic includes the early modeling of SARS by $[10,11]$ to obtain the all-important basic reproduction number for SARS, [12] on modeling the community and hospital transmission of SARS, and $[13,14]$ on models for data-fitting of SARS in Taiwan. Also see [15] for a review of mathematical models of SARS. Recent modeling work of epidemics with intervention measures (quarantine, vaccination, evacuation, etc.) includes $[16,17,18,19,20]$ on smallpox, [17] on flu, [21] on bubonic plague, [22] on measles and whooping cough, [23] on optimal intervention strategies, and 
[18] on a class of infectious disease models with quarantine.

This article is organized as follows: In section 2 we describe the general model with the intervention measures to be considered and the computation of basic reproduction numbers. In section 3 we give the complete analysis of the model with severitydependent public response but without quarantine. Section 4 gives analytical results for the full model with quarantine and full description of its dynamics. Finally in section 5 we discuss the biological significance of our results.

2. The model. In this work, we propose a general model with Level A and B quarantines, as well as imported cases who entered the exposed class upon their arrival before April 28, but were quarantined (Level B) as they entered from the affected areas after April 28. The model variables are given as follows; note that the time unit is in days:

$S$ - the number of susceptible individuals at time $t$;

$E$ - the number of infected asymptomatic persons at time $t$;

$Q_{A}$ - the number of asymptomatic infected persons at time $t$ under Level A quarantine;

$Q_{B}$-the number of imported asymptomatic infected persons at time $t$ (who were under B quarantine if arriving from affected areas after April 28);

$I$ - the number of infective persons with onset of symptoms not isolated or quarantined at time $t$;

$P$ - the number of isolated probable SARS cases at time $t$;

$D$ - the cumulative number of SARS deaths at time $t$;

$R$ - the cumulative number of discharged SARS patients at time $t$.

The key assumptions used are as follows:

1. A SARS-infected person is infective after onset of symptoms.

2. A quarantined person is quarantined without symptoms (hence is not infective), becoming infective with reduced contact rates due to quarantine, and is isolated upon diagnosis.

3. An infected person can infect others unless quarantined or isolated as a probable case with reduced contact rate depending on the effectiveness of the isolation. The underlying assumption here is that once diagnosed as a probable SARS case and hospitalized, a patient cannot infect others.

4. A probable case is removed from isolation either by death or discharge.

5. As behavior change by individuals occurs as a result of public response to the severity of the outbreak, the infection rate (or the product of transmission probability and contact rate) decreases with the increasing cumulative number of probable cases. Similarly, the effectiveness of quarantine and isolation also increases with the increasing number of probable cases, resulting in a decreased number of infections. To account for this decrease, we make use of a rational function $\frac{1}{1+a[P(t)+R(t)+D(t)]}$, where $P+R+D$ is the cumulative number of probable cases. We note that the decreasing rational function used, which resembles Holling's functional response in predator-prey models [24], is not the only choice of function to portray the phenomenon in question. A decreasing exponential function, for example, could do just as well.

6 . We assume homogeneous mixing with quarantine-adjusted incidence.

7. Quarantine for Level A is proportionate to the number of infected asymptomatic persons.

8. Imported cases are a function of time $(Q(t)=0,1$, or 2 as deduced from data), with Level B quarantine after April 28.

The model parameters are as follows: 
$\lambda$-infection rate due to contact with infective class;

$q_{1}$ - proportion of recruitment of asymptomatic infected persons for Level A quarantine;

$\gamma_{3}$-isolation rate of infectives not under quarantine;

$\mu$ - progression rate from exposure to onset of symptoms;

$\gamma_{i}, i=1,2$-isolation rates of $Q_{A}$ and $Q_{B}$, respectively;

$\alpha_{A}, \alpha_{B}, \alpha_{P}$-the proportionate reduction in infectivity of quarantined persons due to Level A and B quarantines (before isolation) and probable cases, respectively; $\rho_{i}, i=1,2$-respective fatality rates of infective cases and isolated probable SARS patients;

$\sigma_{i}, i=1,2$-respective discharge rates of infective cases and isolated probable SARS patients;

$c$ - contact rate in absence of an outbreak;

$a$ - the effect of behavior change in reduction of contact due to the cumulative number of probable cases;

$\beta$ - transmission probability per effective contact.

The flowchart for the model is given in Figure 2.1.

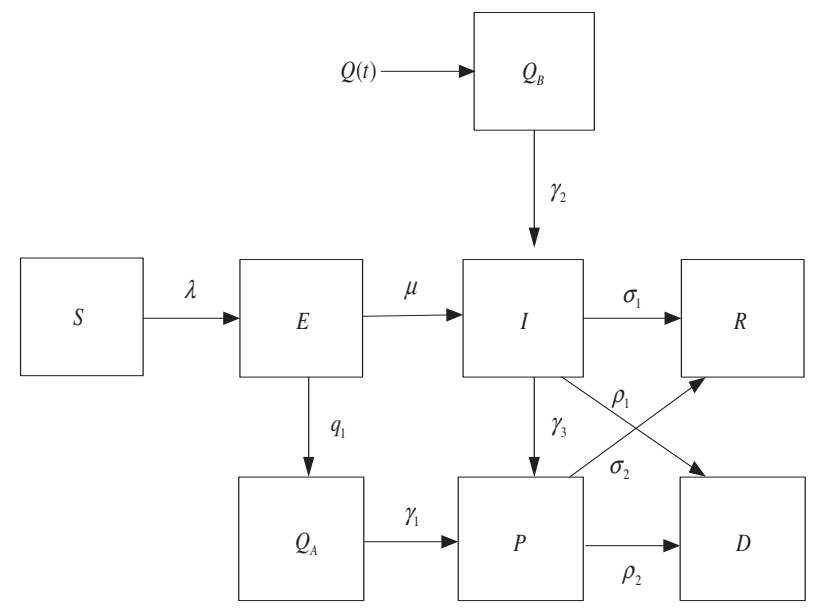

FIG. 2.1. Flowchart for the model.

Finally, the model equations with imported cases, Level A and B quarantines, and behavior change are as follows:

$$
\begin{aligned}
S^{\prime} & =-\lambda\left(S, E, I, Q_{A}, Q_{B}, P, R, D\right) S, \\
E^{\prime} & =\lambda\left(S, E, I, Q_{A}, Q_{B}, P, R, D\right) S-\mu E-q_{1} E, \\
Q_{A}^{\prime} & =q_{1} E-\gamma_{1} Q_{A}, \\
Q_{B}^{\prime} & =Q(t)-\gamma_{2} Q_{B}, \\
I^{\prime} & =\mu E+\gamma_{2} Q_{B}-\left(\sigma_{1}+\rho_{1}+\gamma_{3}\right) I, \\
P^{\prime} & =\gamma_{1} Q_{A}+\gamma_{3} I-\left(\sigma_{2}+\rho_{2}\right) P, \\
R^{\prime} & =\sigma_{1} I+\sigma_{2} P, \\
D^{\prime} & =\rho_{1} I+\rho_{2} P,
\end{aligned}
$$

where the incidence of infection with quarantine is given by 


$$
\begin{aligned}
\lambda\left(S, E, I, Q_{A}, Q_{B}, P, R, D\right)= & \beta \frac{c}{1+a(P+R+D)} \\
& \times \frac{I+\alpha_{A} Q_{A}+\alpha_{B} Q_{B}+\alpha_{P} P}{S+E+I+\alpha_{A} Q_{A}+\alpha_{B} Q_{B}+\alpha_{P} P} .
\end{aligned}
$$

Note that $S+E+Q_{A}+Q_{B}+I+P+R+D=S_{0}+I_{0}$, where $S_{0}$ and $I_{0}$ are the initial susceptible population sizes. The system is nonautonomous, due to the imported case term $Q(t)$ in the right-hand side of the equation for $Q_{B}^{\prime}$. Brauer and van den Driessche [25] have shown that, if there is a positive flow of infectives into the population, disease-free equilibrium might not exist. However, during the SARS outbreak border control was implemented in Taiwan as well as all other affected areas. Therefore we can reasonably assume that $Q(t)$ has compact support and subsequently the asymptotic properties of the nonautonomous system given in (2.1)-(2.8) are the same as the corresponding autonomous system, i.e., with $Q(t)=0$. Hence we need only consider the autonomous system hereafter.

For the model without quarantine, the model equations become

$$
\begin{aligned}
S^{\prime} & =-\lambda(S, E, I, P, D) S, \\
E^{\prime} & =\lambda(S, E, I, P, D) S-\mu E, \\
I^{\prime} & =\mu E-\left(\sigma_{1}+\rho_{1}+\gamma_{3}\right) I, \\
P^{\prime} & =\gamma_{3} I-\left(\sigma_{2}+\rho_{2}\right) P, \\
R^{\prime} & =\sigma_{1} I+\sigma_{2} P, \\
D^{\prime} & =\rho_{1} I+\rho_{2} P,
\end{aligned}
$$

where

$$
\lambda(S, E, I, P, R, D)=\beta\left[\frac{c}{1+a(P+R+D)}\right] \frac{I+\alpha_{P} P}{S+E+I+\alpha_{P} P} .
$$

The disease-free equilibrium (DFE) for the six-dimensional system in $(S, E, I, P, R, D)$ is $\left(S^{*}, 0,0,0, R^{*}, D^{*}\right)$ with $S^{*}+R^{*}+D^{*}=S_{0}+I_{0}$; the endemic equilibrium is $\left(0,0,0,0, R^{\#}, D^{\#}\right)$ with $R^{\#}+D^{\#}=S_{0}+I_{0}$.

Making use of the method in [26], we obtain the expression for the basic reproduction number $R_{0}$ of this case:

$$
\begin{aligned}
R_{0}= & \frac{\beta c}{\left(\sigma_{1}+\rho_{1}+\gamma_{3}\right)\left[1+a\left(R^{*}+D^{*}\right)\right]} \\
& +\frac{\beta c \alpha_{P} \gamma_{3}}{\left(\sigma_{1}+\rho_{1}+\gamma_{3}\right)\left[1+a\left(R^{*}+D^{*}\right)\right]\left(\sigma_{2}+\rho_{2}\right)} .
\end{aligned}
$$

Similarly as for the original model with quarantine, we have the more general expression for the effective basic reproduction number with quarantine $R_{Q}$, again using the procedure developed in [26]:

$$
\begin{aligned}
R_{Q}= & \beta \frac{c}{\left[1+a\left(R^{*}+D^{*}\right)\right]}\left\{\frac{\mu}{\left(\sigma_{1}+\rho_{1}+\gamma_{3}\right)\left[\mu+q_{1}\right]}+\frac{\alpha_{A} q_{1}}{\gamma_{1}\left[\mu+q_{1}\right]}\right\} \\
& +\beta \frac{c}{\left[1+a\left(R^{*}+D^{*}\right)\right]} \frac{\alpha_{P}}{\left(\sigma_{2}+\rho_{2}\right)}\left\{\frac{\gamma_{3}}{\left(\sigma_{1}+\rho_{1}+\gamma_{3}\right)} \frac{\mu}{\left[\mu+q_{1}\right]}+\frac{q_{1}}{\mu+q_{1}}\right\} .
\end{aligned}
$$

Note that both $R_{0}$ and $R_{Q}$ have very clear biological interpretations which will be discussed in section 5 . 
3. Analysis for model without quarantine. In this section we provide full analysis for the model without quarantine. To simplify, we let $\alpha_{p}=0$. That is, hospitalized and isolated probable cases do not make a significant contribution to the infections, as indicated by the result in a data-motivated modeling study of Taiwan's SARS outbreak in [27]. We note that while it is true that nosocomial infections played a crucial role during the SARS outbreak in all affected, as confirmed by the fact that nearly $80 \%$ of SARS infections in Taiwan occurred nosocomially [28], most had occurred before the infective individuals had been diagnosed with SARS and hospitalized with adequate isolation. Only a small number of infections in Taiwan as well as in other affected areas have been documented as being caused by a confirmed or probable SARS patient who most likely had been isolated.

Hence the system in (2.9)-(2.14) becomes

$$
\begin{aligned}
S^{\prime} & =-\frac{\beta I S}{E+I+S} \frac{c}{1+a(P+R+D)}, \\
E^{\prime} & =\frac{\beta I S}{E+I+S} \frac{c}{1+a(P+R+D)}-\mu E, \\
I^{\prime} & =\mu E-\left(\sigma_{1}+\rho_{1}+\gamma_{3}\right) I \\
P^{\prime} & =\gamma_{3} I-\left(\sigma_{2}+\rho_{2}\right) P \\
R^{\prime} & =\sigma_{1} I+\sigma_{2} P \\
D^{\prime} & =\rho_{1} I+\rho_{2} P
\end{aligned}
$$

with $S(0)=S_{0}>0, I(0)=I_{0}>0, E(0)=P(0)=R(0)=D(0)=0$.

We first give the following lemma, the proof of which is in [29].

LEMma 3.1. Let $f$ be continuously differentiable. If $f(t) \longrightarrow$ constant as $t \rightarrow \infty$ and $\left|f^{\prime \prime}(t)\right| \leq M$ for all $t$, then $f^{\prime}(t) \rightarrow 0$ as $t \rightarrow \infty$.

THEOREM 3.2. We have the following asymptotic properties: $S(t) \longrightarrow S_{\infty} \geq 0$, $R(t) \longrightarrow R_{\infty}>0, D(t) \longrightarrow D_{\infty}>0$, and $\lim _{t \rightarrow \infty} I(t)=0, \lim _{t \rightarrow \infty} E(t)=0$, $\lim _{t \rightarrow \infty} P(t)=0$.

Proof. Obviously $S(t)$ is monotone decreasing and bounded below; hence $\lim _{t \rightarrow \infty}$ $S(t)=S_{\infty} \geq 0,0 \leq S_{\infty}<S_{0}$. Moreover, $(E+S)^{\prime}=-\mu E$, and therefore $E(t)+S(t)$ is monotone decreasing for $t \geq 0$. Hence $E(t) \longrightarrow E_{\infty} \geq 0$ as $t \rightarrow \infty$. Since $S(t)+E(t)+I(t)+P(t)+R(t)+D(t) \equiv N=S_{0}+I_{0}$ for all $t, R^{\prime} \geq 0, D^{\prime} \geq 0 \Longrightarrow$ $R(t) \longrightarrow R_{\infty}>0, D(t) \longrightarrow D_{\infty}>0$. Obviously $R^{\prime \prime}=\sigma_{1} I^{\prime}+\sigma_{2} P^{\prime},\left|I^{\prime}\right|$ and $\left|P^{\prime}\right|$ are bounded, and hence $\left|R^{\prime \prime}\right| \leq M$ for some $M>0$. Consequently by Lemma 3.1 $I(t) \longrightarrow I_{\infty}=0, P(t) \longrightarrow P_{\infty}=0$ as $t \rightarrow \infty$.

Claim: $E_{\infty}=0$. Suppose $E_{\infty}>0$; then $I^{\prime}=\mu E-\left(\sigma_{1}+\rho_{1}+\gamma_{3}\right) I \geq \mu\left(E_{\infty}-\varepsilon\right)-$ $\left(\sigma_{1}+\rho_{1}+\gamma_{3}\right) \varepsilon>0$ for $\varepsilon$ small, $t$ large. It follows that $I(t)$ becomes unbounded. This is a contradiction.

Next, we let $q=\sigma_{1}+\rho_{1}+\gamma_{3}$ and $c=1$ (i.e., $\beta$ denotes contact rate times transmission probability) for the sake of simplicity. We also, for the moment, assume $a=0$, i.e., no behavior change. We will return to discuss the case with behavior change later in this section. Subsequently, we consider the following simplified system:

$$
\begin{aligned}
S^{\prime} & =-\frac{\beta I S}{E+I+S}, \\
E^{\prime} & =\frac{\beta I S}{E+I+S}-\mu E, \\
I^{\prime} & =\mu E-q I,
\end{aligned}
$$


with $S(0)=S_{0}>0, I(0)=I_{0}>0, E(0)=0$.

Now, we let $W_{1}=S / I, W_{2}=E / I$. Then (3.7)-(3.9) become

$$
\begin{aligned}
& W_{1}^{\prime}=-\frac{\beta W_{1}}{1+W_{1}+W_{2}}-W_{1}\left(\mu W_{2}-q\right), \\
& W_{2}^{\prime}=\frac{\beta W_{1}}{1+W_{1}+W_{2}}-\mu W_{2}-W_{2}\left(\mu W_{2}-q\right),
\end{aligned}
$$

with $W_{1}(0)>0, W_{2}(0)=0$.

To study the flow of (3.10)-(3.11) in the $W_{2} W_{1}$-phase plane, we first consider the isoclines $W_{1}^{\prime}=0$ and $W_{2}^{\prime}=0$. Clearly, $W_{1}^{\prime}<0$ if $W_{2}>q / \mu$. Moreover,

$$
\begin{aligned}
W_{1}^{\prime} \geq 0 & \Longleftrightarrow q-\mu W_{2} \geq \frac{\beta}{1+W_{1}+W_{2}} \\
& \Longleftrightarrow 1+W_{1}+W_{2} \geq \frac{\beta}{q-\mu W_{2}} \quad \text { for } \quad q-\mu W_{2} \geq 0 \\
& \Longleftrightarrow W_{1} \geq \frac{\beta}{q-\mu W_{2}}-\left(1+W_{2}\right)=f\left(W_{2}\right) \text { for } q-\mu W_{2} \geq 0,
\end{aligned}
$$

and

$$
\begin{aligned}
W_{2}^{\prime} \geq 0 & \Longleftrightarrow \frac{\beta W_{1}}{1+W_{1}+W_{2}} \geq\left[\mu+\left(\mu W_{2}-q\right)\right] W_{2} \\
& \Longleftrightarrow \beta W_{1} \geq\left[\mu+\left(\mu W_{2}-q\right)\right] W_{2}\left(1+W_{1}+W_{2}\right) \\
& \Longleftrightarrow \beta-W_{2}\left[\mu+\left(\mu W_{2}-q\right)\right] W_{1}>\left[\mu+\left(\mu W_{2}-q\right)\right] W_{2}\left(1+W_{2}\right) \\
& \Longleftrightarrow W_{1}>\frac{W_{2}\left(1+W_{2}\right)\left[\mu+\left(\mu W_{2}-q\right)\right]}{\beta-W_{2}\left[\mu+\left(\mu W_{2}-q\right)\right]}=g\left(W_{2}\right) .
\end{aligned}
$$

Consequently, it is easy to verify that the curves $W_{1}=f\left(W_{2}\right)$ and $W_{1}=g\left(W_{2}\right)$ do not intersect.

There are four cases to be considered:

1. $q<\beta, q<\mu$.

Let $\widetilde{W}_{2}$ be the positive root of

$$
h\left(W_{2}\right)=\beta-W_{2}\left[\mu+\left(\mu W_{2}-q\right)\right]=0 .
$$

Clearly $h\left(\frac{q}{\mu}\right)=\beta-q>0$. Hence $\frac{q}{\mu}<\widetilde{W_{2}}$. In the first quadrant of the $W_{2} W_{1}$-phase plane, the isocline $W_{1}=0,\left(W_{1}=f\left(W_{2}\right)\right) 0 \leq W_{2}<\frac{q}{\mu}$, satisfies $f(0)=\frac{\beta}{q}-1>0$ and $f\left(\left(\frac{q}{\mu}\right)^{-}\right)=\infty$. The isocline $\dot{W}_{2}=0\left(W_{1}=g\left(W_{2}\right)\right)$ satisfies $g(0)=0, g\left(\widetilde{W}_{2}-\right)=\infty$. We note that the isocline $\dot{W}_{1}=0$ is above that of $\dot{W}_{2}=0$. Every trajectory converges to the endemic equilibrium $(S / I, E / I)=(0,0)$ as $t \rightarrow \infty$.

2. $\beta>q, \mu<q$.

There are two equilibria $(0,0)$ and $\left(W_{2}^{*}, 0\right)$, where $W_{2}^{*}=\frac{q}{\mu}-1$. Similar to case 1 , every trajectory converges to $\left(W_{2}^{*}, 0\right)$.

3. $\beta<q, \mu<q$.

Clearly $f\left(W_{2}^{*}\right)=f\left(\frac{q}{\mu}-1\right)=\frac{\beta}{\mu}-\frac{q}{\mu}<0$. Observe that $h\left(\frac{q}{\mu}\right)=\beta-q<0$ and we have $\widetilde{W}_{2}<\frac{q}{\mu}$. Since the isocline $\dot{W}_{2}=0$ is above that of $\dot{W}_{1}=0$, it follows that, as $t \longrightarrow \infty, h\left(W_{2}(t)\right) \longrightarrow \widetilde{W_{2}}$, the positive root of $h\left(W_{2}\right)=0$, and $W_{1}(t) \longrightarrow \infty$. Moreover, $\left(W_{1}(t), W_{2}(t)\right)$ approaches the curve $W_{1}=g\left(W_{2}\right)$, i.e., $W_{2}=0$, as $t \rightarrow \infty$. 
4. $\beta<q, q<\mu$.

Obviously, this case is similar to the previous case with $W_{2}(t) \rightarrow \widetilde{W_{2}}$ and $W_{1}(t) \rightarrow \infty$ as $t \rightarrow \infty$.

We then have the following theorem.

TheOREm 3.3. For system (3.7)-(3.9), if $\beta>q$, then $S(t) \rightarrow 0$ as $t \rightarrow \infty$. If $\beta<q$, then $S(t) \rightarrow S_{\infty}>0$ as $t \rightarrow \infty$.

Proof. For cases 1 and 2 as described earlier, $\frac{S(t)}{I(t)}=W_{1}(t) \rightarrow 0$ as $t \rightarrow \infty$. Since $I(t) \longrightarrow 0$ and $S(t) \longrightarrow S_{\infty} \geq 0$, we have $S_{\infty}=0$. Hence, $\beta>q$ implies $S(t) \longrightarrow 0$ as $t \rightarrow \infty$. If $\beta<q$, then, by cases 3 and 4 , we have $W_{1}(t) \longrightarrow \infty$ and $W_{2}(t) \longrightarrow \widetilde{W}_{2}>0$ as $t \rightarrow \infty$.

Claim: $S_{\infty}>0$. If $S_{\infty}=0$, then

$\int_{0}^{\infty} \frac{1}{1+W_{1}(t)+W_{2}(t)} d t=\infty \quad$ and $\quad \int_{0}^{\infty} \frac{1}{W_{1}(t)} d t \geq \int_{0}^{\infty} \frac{1}{1+W_{1}(t)+W_{2}(t)} d t=\infty$.

Therefore we have $\int_{0}^{\infty} \frac{1}{W_{1}(t)} d t=\infty$.

From (4.10),

$$
W_{1}^{\prime}=\frac{-\beta W_{1}}{1+W_{1}+W_{2}}-W_{1}\left(\mu W_{2}-q\right) \geq \frac{-\beta W_{1}}{1+W_{1}+\widetilde{W}_{2}-\epsilon}+W_{1}\left[q-\mu\left(\widetilde{W_{2}}+\epsilon\right)\right],
$$

where $\widetilde{W_{2}}-\epsilon<W_{2}(t)<\widetilde{W_{2}}+\epsilon$ for $t \geq t_{0}$.

For $t \geq t_{0}$,

$$
\frac{W_{1}^{\prime}}{W_{1}} \geq \frac{-\beta}{1+W_{1}+\widetilde{W}_{2}-\epsilon}+\left[q-\mu\left(\widetilde{W}_{2}+\epsilon\right)\right]
$$

Because $W_{1}(t) \rightarrow \infty$ as $t \rightarrow \infty$,

$$
\frac{W_{1}^{\prime}}{W_{1}} \geq \frac{1}{2}\left[q-\mu\left(\widetilde{W_{2}}+\epsilon\right)\right]>0 \quad \text { for } t \geq T, \text { for some } T \text { large. }
$$

Therefore $W_{1} \rightarrow \infty$ exponentially, and

$$
W_{1}(t) \geq W_{1}(T) \exp \left\{\frac{1}{2}\left[q-\mu\left(\widetilde{W}_{2}+\epsilon\right)\right](t-T)\right\} .
$$

But

$$
\infty=\int_{T}^{\infty} \frac{1}{W_{1}(t)} d t \leq \int_{T}^{\infty} \frac{1}{W_{1}(T) \exp \left\{\frac{1}{2}\left[q-\mu\left(\widetilde{W_{2}}+\epsilon\right)(t-T)\right]\right\}} d t<\infty .
$$

This is a contradiction.

Now we return to consider system (3.1)-(3.6) with behavior change.

Theorem 3.4. Let $\widetilde{\beta}=\frac{\beta c}{1+a N}$, where $N=R_{\infty}^{*}+D_{\infty}^{*}$.

(i) If $q=\sigma_{1}+\rho_{1}+\gamma_{3}>\widetilde{\beta}$, then the solution of system (3.1)-(3.6) satisfies $S(t) \longrightarrow S_{\infty}>0$ as $t \rightarrow \infty$.

(ii) If $q<\widetilde{\beta}$, then $S(t) \longrightarrow 0$ as $t \rightarrow \infty$.

Note that the condition $q<\widetilde{\beta}$ in the above theorem is equivalent to $R_{0}>1$ with $R_{0}$ as defined in (2.15) and $\alpha_{P}=0$. Hence with this theorem we have shown that the asymptotic result for $R_{0}$ is global. 
Proof. (i) Suppose not; then $\lim _{t \rightarrow \infty} S(t)=S_{\infty}^{*}=0$. Consider the limiting system of

$$
\begin{aligned}
S^{\prime} & =-\frac{\beta I S}{E+I+S} \frac{c}{1+a(P+R+D)} a \geq 0, \\
E^{\prime} & =\frac{\beta I S}{E+I+S} \frac{c}{1+a(P+R+D)}-\mu E, \\
I^{\prime} & =\mu E-\left(\sigma_{1}+\rho_{1}+\gamma_{3}\right) I .
\end{aligned}
$$

Since $P(t) \longrightarrow 0, R(t) \longrightarrow R_{\infty}^{*}$, and $D(t) \longrightarrow D_{\infty}^{*}$ as $t \rightarrow \infty$, we have the limiting system as follows:

$$
\begin{aligned}
S^{\prime} & =-\frac{\beta I S}{E+I+S} \frac{c}{1+a N}, \quad a \geq 0, \\
E^{\prime} & =\frac{\beta I S}{E+I+S} \frac{c}{1+a N}-\mu E, \\
I^{\prime} & =\mu E-\left(\sigma_{1}+\rho_{1}+\gamma_{3}\right) I .
\end{aligned}
$$

From the analysis of system (3.7)-(3.9) with

$$
\widetilde{\beta}=\frac{\beta c}{1+a N},
$$

if $q>\widetilde{\beta}$, then $S(t) \rightarrow S_{\infty}>0$. This is a contradiction.

(ii) Assuming $q<\widetilde{\beta}$, we want to show $\lim _{t \rightarrow \infty} S(t)=0$. If not, then $\lim _{t \rightarrow \infty} S(t)$ $=S_{\infty}>0$. In this case, we have $R_{\infty}+D_{\infty}+S_{\infty}=N$, where $R_{\infty}=\lim _{t \rightarrow \infty} R(t)$, $D_{\infty}=\lim _{t \rightarrow \infty} D(t)$.

Since $S_{\infty}>0, D_{\infty}+R_{\infty}<N$. The limiting system of (3.12)-(3.14) is system (3.15)-(3.17) with $D_{\infty}^{*}, R_{\infty}^{*}$ replaced by $D_{\infty}, R_{\infty}$. From the analysis of system (3.7)-(3.9) and the assumption $q<\widetilde{\beta}$, we have

$$
\widehat{\beta}=\frac{c \beta}{1+a\left(D_{\infty}+R_{\infty}\right)}>\frac{c \beta}{1+a N}=\widetilde{\beta}>q ;
$$

hence $S(t) \longrightarrow 0$ as $t \rightarrow \infty$. This is a contradiction.

4. Analysis for model with quarantine. We now give some analytical results on the model with Level A quarantine only. We then have the system

$$
\begin{aligned}
S^{\prime} & =-\lambda\left(S, E, I, Q_{A}, P, R, D\right) S, \\
E^{\prime} & =\lambda\left(S, E, I, Q_{A}, P, R, D\right) S-\mu E-q_{1} E, \\
Q_{A}^{\prime} & =q_{1} E-\gamma_{1} Q_{A}, \\
I^{\prime} & =\mu E-\left(\sigma_{1}+\rho_{1}+\gamma_{3}\right) I, \\
P^{\prime} & =\gamma_{1} Q_{A}+\gamma_{3} I-\left(\sigma_{2}+\rho_{2}\right) P, \\
R^{\prime} & =\sigma_{1} I+\sigma_{2} P, \\
D^{\prime} & =\rho_{1} I+\rho_{2} P,
\end{aligned}
$$

where the incidence of infection with quarantine rates is given by

$$
\lambda\left(S, E, I, Q_{A}, P, R, D\right)=\beta \frac{I+\alpha_{A} Q_{A}}{S+E+I+\alpha_{A} Q_{A}} \frac{c}{1+a(P+R+D)} .
$$


Again $S+E+Q_{A}+I+P+R+D=S_{0}+I_{0} \equiv N$, where $S(0)=S_{0}>0, I(0)=I_{0}>0$.

As in Theorem 3.2, we have $S(t) \longrightarrow S_{\infty} \geq 0, R(t) \longrightarrow R_{\infty}>0, D(t) \longrightarrow D_{\infty}>$ 0 and $I(t) \longrightarrow 0, E(t) \longrightarrow 0, P(t) \longrightarrow 0, Q_{A}(t) \longrightarrow 0$ as $t \longrightarrow \infty$.

Next we let $\widetilde{q}=\sigma_{1}+\rho_{1}+\gamma_{3}, c=1$, and $a=0$ and consider the limiting system

$$
\begin{aligned}
S^{\prime} & =\frac{-\beta\left(I+\alpha_{A} Q_{A}\right)}{S+E+I+\alpha_{A} Q_{A}} S, \\
E^{\prime} & =\frac{\beta\left(I+\alpha_{A} Q_{A}\right)}{S+E+I+\alpha_{A} Q_{A}} S-\mu E-q_{1} E, \\
Q_{A}^{\prime} & =q_{1} E-\gamma_{1} Q_{A}, \\
I^{\prime} & =\mu E-\widetilde{q} I .
\end{aligned}
$$

Let $W_{1}=\frac{S}{I}, W_{2}=\frac{E}{I}, W_{3}=\frac{Q_{A}}{I}$. Then we have

$$
\begin{aligned}
W_{1}^{\prime} & =-\frac{\beta\left(1+\alpha_{A} W_{3}\right)}{1+W_{1}+W_{2}+\alpha_{A} W_{3}} W_{1}-\left(\mu W_{2}-\widetilde{q}\right) W_{1}, \\
W_{2}^{\prime} & =\frac{\beta\left(1+\alpha_{A} W_{3}\right)}{1+W_{1}+W_{2}+\alpha_{A} W_{3}} W_{1}-\left(\mu+q_{1}\right) W_{2}-\left(\mu W_{2}-\widetilde{q}\right) W_{2}, \\
W_{3}^{\prime} & =\left(q_{1} W_{2}-\gamma_{1} W_{3}\right)-\left(\mu W_{2}-\widetilde{q}\right) W_{3} .
\end{aligned}
$$

We note that if $\alpha_{A}=0, q_{1}>0$, then (4.10) is reduced to the two-dimensional system

$$
\begin{aligned}
& W_{1}^{\prime}=\frac{-\beta W_{1}}{1+W_{1}+W_{2}}-\left(\mu W_{2}-\widetilde{q}\right) W_{1}, \\
& W_{2}^{\prime}=\frac{\beta W_{1}}{1+W_{1}+W_{2}}-\left(\mu+q_{1}\right) W_{2}-\left(\mu W_{2}-\widetilde{q}\right) W_{2} .
\end{aligned}
$$

As in Theorem 3.4, it can be shown that (i) if $\widetilde{\beta}<\widetilde{q}\left(\frac{\mu+q_{1}}{\mu}\right)$, then $S(t) \rightarrow S_{\infty}>0$ as $t \rightarrow \infty$ and (ii) if $\widetilde{\beta}>\widetilde{q}\left(\frac{\mu+q_{1}}{\mu}\right)$, then $S(t) \rightarrow 0$ as $t \rightarrow \infty$.

We give the following equilibria and their respective stability analyses:

1. $E_{0}=(0,0,0)$ is an equilibrium of (4.10). Then the variational matrix at $E_{0}$ is

$$
M_{0}=\left[\begin{array}{ccc}
-\beta+\widetilde{q} & 0 & 0 \\
\beta & -\left(\mu+q_{1}\right)+\widetilde{q} & 0 \\
0 & q_{1} & -\gamma_{1}+\widetilde{q}
\end{array}\right] .
$$

We then have the following trivial lemma.

Lemma 4.1. $E_{0}=(0,0,0)$ is locally asymptotically stable if $\beta>\widetilde{q}, \mu+q_{1}>\widetilde{q}$, $\gamma_{1}>\widetilde{q}$.

2. $E_{23}^{*}=\left(0, W_{2}^{*}, W_{3}^{*}\right)$, where

$$
\begin{aligned}
& W_{2}^{*}=\frac{\widetilde{q}-\left(\mu+q_{1}\right)}{\mu}>0 \Longleftrightarrow \widetilde{q}>\mu+q_{1}, \\
& W_{3}^{*}=\frac{q_{1} W_{2}^{*}}{\left(\mu W_{2}^{*}-\widetilde{q}\right)+\gamma_{1}}>0 \Longleftrightarrow \gamma_{1}>\mu+q_{1} .
\end{aligned}
$$

Note that $E_{23}^{*}=\left(0, W_{2}^{*}, W_{3}^{*}\right)$ exists if $\widetilde{q}>\mu+q_{1}, \gamma_{1}>\mu+q_{1}$. 
The variational matrix at $E_{23}^{*}$ is

$$
M^{*}=\left[\begin{array}{lll}
\frac{-\beta\left(1+\alpha_{A} W_{3}^{*}\right)}{1+W_{2}^{*}+\alpha_{A} W_{3}^{*}}-\left(\mu W_{2}^{*}-q\right) & 0 & 0 \\
\frac{\beta\left(1+\alpha_{A} W_{3}^{*}\right)}{1+W_{2}^{*}+\alpha_{A} W_{3}^{*}} & -\left(\mu+q_{1}\right)-\left(2 \mu W_{2}^{*}-\widetilde{q}\right) & 0 \\
0 & q_{1}-\mu W_{3}^{*} & -\gamma_{1}-\left(\mu W_{2}^{*}-\widetilde{q}\right)
\end{array}\right] .
$$

The local stability result is given below, the proof of which is also trivial.

LEMMA 4.2. $E_{23}^{*}$ is locally asymptotically stable if $\beta>\frac{1+W_{2}^{*}+\alpha_{A} W_{3}^{*}}{1+\alpha_{A} W_{3}^{*}}\left(\mu+q_{1}\right)$.

3. $E_{\infty}=\left(+\infty, \widetilde{W_{2}}, \widetilde{W}_{3}\right)$, where $\widetilde{W}_{2}<\frac{q}{\mu}$. From the first equation of $(4.10)$, we have $\frac{W_{1}^{\prime}}{W_{1}} \leq-\left(\mu W_{2}-\widetilde{q}\right)$. If $\lim _{t \rightarrow \infty} W_{1}(t)=\infty$, then we must have $\widetilde{W}_{2}<\frac{q}{\mu}$. From the second and third equations of (4.10) and $\lim _{t \rightarrow \infty} W_{1}(t)=\infty$, it can be shown that $\left(\widetilde{W_{2}}, \widetilde{W}_{3}\right)$ is the solution of

$$
\begin{aligned}
\beta\left(1+\alpha_{A} W_{3}\right)-\left(\mu W_{2}-\widetilde{q}\right) W_{2}-\left(\mu+q_{1}\right) W_{2} & =0, \\
\left(q_{1} W_{2}-\gamma_{1} W_{3}\right)-\left(\mu W_{2}-\widetilde{q}\right) W_{3} & =0 .
\end{aligned}
$$

$$
\begin{aligned}
& Z_{1}^{\prime}=\beta Z_{1} \frac{Z_{1}+\alpha_{A} Z_{3}}{1+Z_{1}+Z_{2}+\alpha_{A} Z_{3}}+\mu Z_{2}-\widetilde{q} Z_{1}, \\
& Z_{2}^{\prime}=\left(1+Z_{2}\right) \frac{\beta\left(Z_{1}+\alpha_{A} Z_{3}\right)}{1+Z_{1}+Z_{2}+\alpha_{A} Z_{3}}-\left(\mu+q_{1}\right) Z_{2}, \\
& Z_{3}^{\prime}=q_{1} Z_{2}-\gamma_{1} Z_{3}+Z_{3} \frac{\beta\left(Z_{1}+\alpha_{A} Z_{3}\right)}{1+Z_{1}+Z_{2}+\alpha_{A} Z_{3}} .
\end{aligned}
$$

The local stability of $E_{\infty}$ for system (4.10) is equivalent to the local stability of $\widehat{E_{0}}=(0,0,0)$ for system (4.11). The variational matrix of $\widehat{E_{0}}$ for system (4.11) is computed as

$$
\left[\begin{array}{ccc}
-\widetilde{q} & \mu & 0 \\
\beta & -\left(\mu+q_{1}\right) & \beta \alpha_{A} \\
0 & q_{1} & -\gamma_{1}
\end{array}\right]
$$

From the Routh-Hurwitz criterion, the stability conditions can be rewritten as

1. $\beta<A_{1}, A_{1}=\frac{\gamma_{1} \widetilde{q}+\left(\mu+q_{1}\right)\left(\gamma_{1}+\widetilde{q}\right)}{\mu+\alpha_{A} \widetilde{q}}$.

2. $\beta<A_{2}, A_{2}=\frac{\left(\mu+q_{1}\right) \gamma_{1} \widetilde{q} q}{\mu \gamma_{1}+\alpha_{A} q_{1} \widetilde{q}}$.

3. $\beta<A_{3}, A_{3}=\frac{\left(\mu+q_{1}\right)^{2}\left(\gamma_{1}+\widetilde{q}\right)+\gamma_{1} \widetilde{q}+\left(\mu+q_{1}\right)\left(\gamma_{1}+\widetilde{q}\right)^{2}}{\mu\left(\mu+q_{1}\right)+\mu \widetilde{q}+\alpha_{A} q_{1}\left(\left(\mu+q_{1}\right)+\gamma_{1}\right)}$.

We now have the following trivial results.

Lemma 4.3. $E_{\infty}$ is stable if $\beta<A_{2}$.

Proof. By routine computation, we have $A_{2}<A_{1}, A_{2}<A_{3}$.

Lemma 4.4. If $\gamma_{1}>\widetilde{q}$, then $A_{2}>\widetilde{q}$.

Proof. Clearly, $A_{2}>\widetilde{q} \Longleftrightarrow \gamma_{1}>\alpha_{A} \widetilde{q}, 0 \leq \alpha_{A} \leq 1$.

Thus, when $\gamma_{1}>\widetilde{q}$, we have the stability of $E_{0}$ and $E_{\infty}$ diagramed as in Figure 4.1.

We note that if $q_{1}=0, \alpha_{A}=0$, then $A_{2}=\widetilde{q}$. If $\alpha_{A}=0$, then $A_{2}=\widetilde{q} \frac{\mu+q_{1}}{\mu}$. If $0<\alpha_{A}<1$, then

$$
A_{2}=\gamma_{1} \widetilde{q} \frac{\mu+q_{1}}{\mu \gamma_{1}+\alpha_{A} q_{1} \widetilde{q}} \rightarrow \frac{\gamma_{1}}{\alpha_{A}} \quad \text { as } q_{1} \longrightarrow \infty
$$




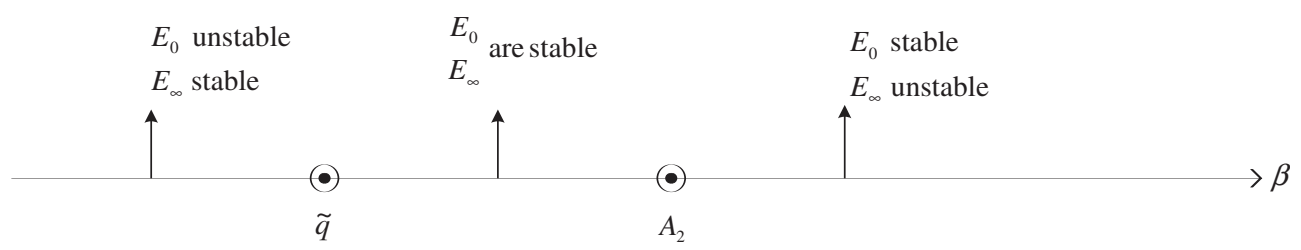

FIG. 4.1. Stability of $E_{0}$ and $E_{\infty}$ when $\gamma_{1}>\widetilde{q}$.

Thus for the model with quarantine measure, there is a region of bistability. The smaller $\alpha_{A}$ and larger $q_{1}$ give a large region of bistability. According to analysis, if the contact rate $\beta<\widetilde{q}$, we would have only the DFE no matter if the quarantine measure is implemented or not. However, if $\beta>A_{2}$, then we have the endemic case, i.e., $S(t) \longrightarrow 0$ as $t \longrightarrow \infty$.

The following lemma is trivial to prove.

Lemma 4.5. If $\mu+q_{1}>\widetilde{q}, \gamma_{1}>\widetilde{q}, \widetilde{q}<\beta<A_{2}$, then both $E_{0}$ and $E_{\infty}$ are locally asymptotically stable. Furthermore, there exists a unique interior equilibrium $E_{c}=\left(W_{1 c}, W_{2 c}, W_{3 c}\right), 0<W_{2 c}<\frac{\widetilde{q}}{\mu}$.

Remark. We conjecture that $E_{c}$ is a saddle point with two-dimensional stable manifold, although we are unable to give a rigorous proof. Instead we will give a full description of the dynamics for the model with quarantine.

Next we consider the case $\widetilde{q}>\mu+q_{1}, \gamma_{1}>\mu+q_{1}$, which guarantees the existence of $E_{23}^{*}$. The following inequality is also easy to obtain.

Lemma 4.6. $A_{2}>\left(\mu+q_{1}\right) \frac{1+W_{2}^{*}+\alpha_{A} W_{3}^{*}}{1+\alpha_{A} W_{3}^{*}}$.

An illustration of the stability of $E_{23}$ and $E_{\infty}$ in this case is given in Figure 4.2.

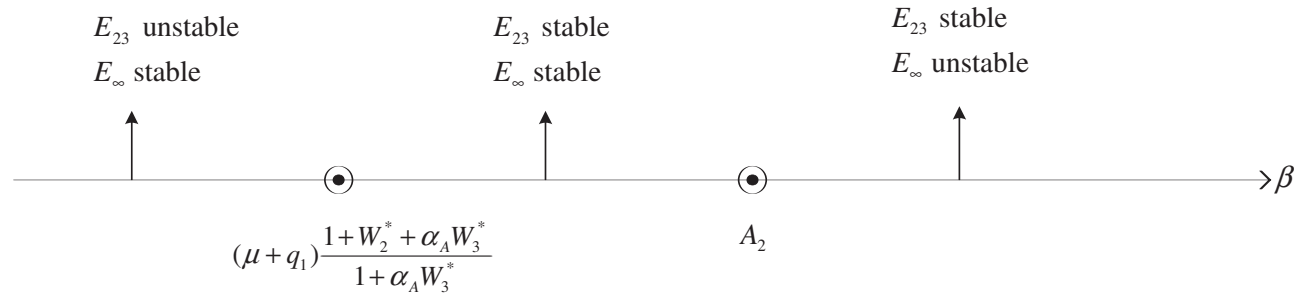

FIG. 4.2. Stability of $E_{23}$ and $E_{\infty}$ when $A_{2}>\left(\mu+q_{1}\right) \frac{1+W_{2}^{*}+\alpha_{A} W_{3}^{*}}{1+\alpha_{A} W_{3}^{*}}$.

We note that if $\alpha_{A}=0, q_{1}=0$, then

$$
\left(\mu+q_{1}\right) \frac{1+W_{2}^{*}+\alpha_{A} W_{3}^{*}}{1+\alpha_{A} W_{3}^{*}}=\widetilde{q} \quad \text { and } \quad A_{2}=\widetilde{q} .
$$

It is also easy to show that

$$
\left(\mu+q_{1}\right) \frac{1+W_{2}^{*}+\alpha_{A} W_{3}^{*}}{1+\alpha_{A} W_{3}^{*}}>\widetilde{q} \Longleftrightarrow \frac{1}{\alpha_{A}}>\frac{\widetilde{q}-\left(\mu+q_{1}\right)}{\gamma_{1}-\left(\mu+q_{1}\right)} .
$$

Thus if $\frac{1}{\alpha_{A}}>\frac{\widetilde{q}-\left(\mu+q_{1}\right)}{\gamma_{1}-\left(\mu+q_{1}\right)}$ (e.g., if $\left.\gamma_{1}>\widetilde{q}\right)$, we have a diagram for the relative sizes of parameters in Figure 4.3.

Thus, for the model with the quarantine measures and the contact rate $\beta, \widetilde{q}<$ $\beta<\left(\mu+q_{1}\right)\left(1+\frac{W_{2}^{*}}{1+\alpha_{A} W_{3}^{*}}\right)$ yields the DFE. On the other hand, we would have the 


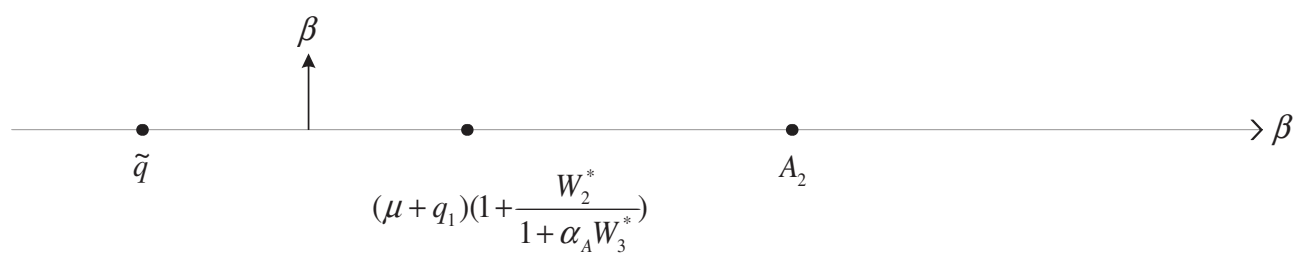

FIG. 4.3. Diagram for the relative sizes of parameters when $\frac{1}{\alpha_{A}}>\frac{\widetilde{q}-\left(\mu+q_{1}\right)}{\gamma_{1}-\left(\mu+q_{1}\right)}\left(\right.$ e.g., if $\left.\gamma_{1}>\widetilde{q}\right)$.

endemic steady state if no quarantine action is taken (i.e., $\beta>\widetilde{q} \Longrightarrow$ endemic steady state in the case of no quarantine). The diagram for the case $\frac{1}{\alpha_{A}}>\frac{\widetilde{q}-\left(\mu+q_{1}\right)}{\gamma_{1}-\left(\mu+q_{1}\right)}$, which is possible if $\gamma_{1}<\widetilde{q}$, has been deleted for brevity.

For the case $\left(\mu+q_{1}\right)\left(1+\frac{W_{2}^{*}}{1+\alpha_{A} W_{3}^{*}}\right)<\beta<\widetilde{q}, E_{23}^{*}$ and $E_{\infty}$ are both stable. Consequently, having quarantine measures implemented might lead to an adverse effect. More precisely, since $\beta<\widetilde{q}$, we have the system approaching DFE when there is no quarantine, but with quarantine, we would have the bistable case where the system could approach an endemic steady state, given that the appropriate initial population is in the stability region of the endemic equilibrium.

Remark. In the bistable case, we again conjecture that the interior equilibrium $\left(E_{c}\right)$ exists and $\left(E_{c}\right)$ is a saddle point with two-dimensional stable manifold.

Now we return to the original system (4.1)-(4.7). Consider the limiting system

$$
\begin{aligned}
S^{\prime} & =-\frac{\beta\left(I+\alpha_{A} Q_{A}\right)}{S+E+I+\alpha_{A} Q_{A}} \frac{c S}{1+a\left(D_{\infty}+R_{\infty}\right)}, \\
E^{\prime} & =\frac{\beta\left(I+\alpha_{A} Q_{A}\right)}{S+E+I+\alpha_{A} Q_{A}} \frac{c S}{1+a\left(D_{\infty}+R_{\infty}\right)}-\left(\mu+q_{1}\right) E, \\
Q_{A}^{\prime} & =q_{1} E-\gamma_{1} Q_{A}, \\
I^{\prime} & =\mu E-\widetilde{q} I .
\end{aligned}
$$

Letting $\widehat{\beta}=\frac{\beta c}{1+a\left(D_{\infty}^{*}+R_{\infty}^{*}\right)}$, we have the following theorem, the proof of which is similar to that of the case without quarantine.

LEMMA 4.7. If $W_{1}(t) \rightarrow \infty$ as $t \rightarrow \infty$, then we have $W_{2}(t) \rightarrow \widetilde{W_{2}}<\infty$, $Z_{3}=\frac{W_{3}}{W_{1}} \rightarrow 0$, and $S(t) \rightarrow S_{\infty}>0$.

We now have the main theorem.

THEOREM 4.8. Let $\widetilde{\beta}=\frac{\beta c}{1+a N}$.

1. If $W_{1}(t) \rightarrow \infty$ as $t \rightarrow \infty, \widetilde{\beta}<A_{2}$, and $E_{0}, E_{23}^{*}$ are unstable, then $S(t) \rightarrow$ $S_{\infty}>0$.

2. If $W_{1}(t) \rightarrow 0$ as $t \rightarrow \infty, \widetilde{\beta}>A_{2}$, and one of the two equilibria, $E_{0}$ or $E_{23}^{*}$, is asymptotically stable, then $S(t) \rightarrow 0$ as $t \rightarrow \infty$.

3. The bistable case occurs when $\widetilde{q}<\widetilde{\beta}<A_{2}$, or $\left(\mu+q_{1}\right)\left(1+\frac{W_{2}^{*}}{1+\alpha_{A} W_{3}^{3}}\right)<\widetilde{\beta}<A_{2}$.

Proof.

1. If not, $S(t) \rightarrow 0$ as $t \rightarrow \infty$, i.e., $S_{\infty}^{*}=0$. Consider the limiting system (4.11) where we have

$$
\widehat{\beta}=\frac{\beta c}{1+a\left(D_{\infty}^{*}+R_{\infty}^{*}\right)}=\frac{\beta c}{1+a N}=\widetilde{\beta}<A_{2} .
$$

It follows that $\lim _{t \rightarrow \infty} W_{1}(t)=+\infty$ (assuming the convergence is global) $\Longrightarrow$ $S(t) \rightarrow S_{\infty}>0$. Hence we have a contradiction. 
2. If not, assume $S(t) \rightarrow S_{\infty}^{*}>0$. Then $S_{\infty}^{*}+D_{\infty}^{*}+R_{\infty}^{*}=N$. Consequently,

$$
\widehat{\beta}=\frac{\beta c}{1+a\left(D_{\infty}^{*}+R_{\infty}^{*}\right)}>\frac{\beta C}{1+a\left(S_{\infty}^{*}+D_{\infty}^{*}+R_{\infty}^{*}\right)}=\frac{\beta C}{1+a N}=\widetilde{\beta}>A_{2}
$$

and $\lim _{t \rightarrow \infty} S(t)=S_{\infty}=0$, again a contradiction.

Remark. It can be shown that the local stability condition for the effective reproduction number with quarantine $R_{Q}<1$ is equivalent to condition 1 of Theorem 4.8, namely, $\widetilde{\beta}=\frac{\beta c}{1+a\left(S_{\infty}^{*}+D_{\infty}^{*}+R_{\infty}^{*}\right)}<A_{2}$.

We further consider the case where $E_{0}=(0,0,0)$ is unstable and $E_{23}^{*}=\left(0, W_{2}^{*}, W_{3}^{*}\right)$ does not exist. We note that $E_{0}=(0,0,0)$ is stable $\Longleftrightarrow \beta>\widetilde{q}, \mu+q_{1}>\widetilde{q}, \gamma_{1}>\widetilde{q}$ and $E_{23}^{*}$ exists $\Longleftrightarrow \mu+q_{1}<\widetilde{q}, \gamma_{1}>\mu+q_{1}$.

We consider the case $W_{3}(t) \rightarrow \infty$ as $t \rightarrow \infty$. Let $U_{1}=W_{1}, U_{2}=W_{2}, U_{3}=\frac{1}{W_{3}}$. Then system (4.10) becomes

$$
\begin{aligned}
U_{1}^{\prime} & =\frac{-\beta\left(U_{3}+\alpha_{A}\right)}{U_{3}\left(1+U_{1}+U_{2}\right)+\alpha_{A}} U_{1}-\left(\mu U_{2}-\widetilde{q}\right) U_{1}, \\
U_{2}^{\prime} & =\frac{\beta\left(U_{3}+\alpha_{A}\right)}{U_{3}\left(1+U_{1}+U_{2}\right)+\alpha_{A}} U_{1}-\left(\mu+q_{1}\right) U_{2}-\left(\mu U_{2}-\widetilde{q}\right) U_{2}, \\
U_{3}^{\prime} & =-q_{1} U_{2} U_{3}^{2}+\gamma_{1} U_{3}+U_{3}\left(\mu U_{2}-\widetilde{q}\right) .
\end{aligned}
$$

We give the equilibria and stability analysis of (4.13):

1. $\widetilde{E_{0}}=(0,0,0)$ always exists.

2. $\widetilde{E_{2}}=\left(0, \widetilde{U}_{2}, 0\right)$, where $\widetilde{U}_{2}$ satisfies $-\left(\mu+q_{1}\right) \widetilde{U}_{2}-\left(\mu \widetilde{U}_{2}-\widetilde{q}\right) \widetilde{U}_{2}=0$. Therefore $\widetilde{U}_{2}=\frac{\widetilde{q}-\left(\mu+q_{1}\right)}{\mu}>0 \Longleftrightarrow \widetilde{q}>\mu+q_{1}$. Subsequently, $\widetilde{E_{2}}$ exists if and only if $\widetilde{q} \geq \mu+q_{1}$.

3. $\widetilde{E_{12}}=\left(U_{1}^{*}, U_{2}^{*}, 0\right)$.

From the first equation of (4.13), $U_{2}^{*}>0$ satisfies $-\beta-\left(\mu U_{2}^{*}-\widetilde{q}\right)=0$. Therefore $U_{2}^{*}=\frac{\widetilde{q}-\beta}{\mu}>0$.

From the second equation of (4.13), $U_{1}^{*}$ satisfies $\beta U_{1}^{*}-\left(\mu+q_{1}\right) U_{2}^{*}-\left(\mu U_{2}^{*}-\right.$ $\widetilde{q}) U_{2}^{*}=0$. Therefore $U_{1}^{*}=\frac{1}{\beta}\left(\left(\mu+q_{1}\right)-\beta\right) U_{2}^{*}>0$.

It follows that $\widetilde{E_{12}}$ exists $\Longleftrightarrow \widetilde{q}>\beta, \mu+q_{1}>\beta$.

(i) Stability of $\widetilde{E_{0}}$. The variational matrix of (4.13) at $\widetilde{E_{0}}$ is

$$
M_{0}=\left[\begin{array}{ccc}
-\beta+\widetilde{q} & 0 & 0 \\
\beta & -\left(\mu+q_{1}\right)+\widetilde{q} & 0 \\
0 & 0 & \gamma_{1}-\widetilde{q}
\end{array}\right] .
$$

Thus $\widetilde{E_{0}}$ is stable $\Longleftrightarrow \widetilde{q}<\beta, \widetilde{q}<\mu+q_{1}, \gamma_{1}<\widetilde{q}$.

(ii) Stability of $\widetilde{E}_{2}$. The variational matrix of (4.13) at $\widetilde{E_{2}}$ is

$$
\left[\begin{array}{ccc}
-\beta+\left(\mu+q_{1}\right) & 0 & 0 \\
\beta & -\widetilde{q}+\left(\mu+q_{1}\right) & 0 \\
0 & 0 & \gamma_{1}-\left(\mu+q_{1}\right)
\end{array}\right] .
$$

Thus $\widetilde{E_{2}}$ is stable $\Longleftrightarrow \mu+q_{1}<\beta, \widetilde{q}>\mu+q_{1}, \gamma_{1}<\mu+q_{1}$.

(iii) Stability of $\widetilde{E_{12}}$. The variational matrix of (4.13) at $\widetilde{E_{12}}$ is

$$
\left[\begin{array}{ccc}
0 & -\mu U_{1}^{*} & * \\
\beta & -\left(\mu+q_{1}\right)-2 \mu U_{2}^{*}+\widetilde{q} & * \\
0 & 0 & \gamma_{1}+\left(\mu U_{2}^{*}-\widetilde{q}\right)
\end{array}\right] .
$$


The eigenvalue $\lambda$ satisfies

$$
\begin{array}{r}
\left(\lambda-\left(\gamma_{1}+\left(\mu U_{2}^{*}-\widetilde{q}\right)\right)\right)\left(\lambda^{2}+\left(\mu+q_{1}+2 \mu U_{2}^{*}-\widetilde{q}\right) \lambda+\beta \mu U_{1}^{*}\right)=0, \\
\gamma_{1}+\mu U_{2}^{*}-\widetilde{q}=\gamma_{1}-\beta \text { implies } \mu+q_{1}+2 \mu U_{2}^{*}-\widetilde{q}=\widetilde{q}+\left(\mu+q_{1}\right)-2 \beta .
\end{array}
$$

Since $\widetilde{E_{12}}$ exists $\Longrightarrow \widetilde{q}>\beta, \mu+q_{1}>\beta$, we have $\mu+q_{1}+2 \mu U_{2}^{*}-\widetilde{q}>0$ and $\widetilde{E_{12}}$ is stable $\Longleftrightarrow \gamma_{1}<\beta, \widetilde{q}>\beta, \mu+q_{1}>\beta$.

Now, when we assume that $E_{0}=(0,0,0)$ is unstable and $E_{23}^{*}$ does not exist, there are three cases:

Case 1. $\widetilde{q}>\mu+q_{1}>\gamma_{1}$, and we have three subcases:

Subcase 1. $A_{2}>\widetilde{q} \Longleftrightarrow \gamma_{1}>\alpha_{A} \widetilde{q}$.

Subcase 2. $A_{2}>\gamma_{1} \Longleftrightarrow\left(\widetilde{q}-\gamma_{1}\right)+\widetilde{q} q_{1}>\alpha_{A} q_{1} \widetilde{q}$.

Subcase 3. $A_{2}>\mu+q_{1} \Longleftrightarrow \gamma_{1}(\widetilde{q}-\mu)>\alpha_{A} q_{1} \widetilde{q}$.

Figure 4.4 illustrates the possibilities for the stability of $\widetilde{E_{12}}$ and $E_{\infty}$ in Case 1.

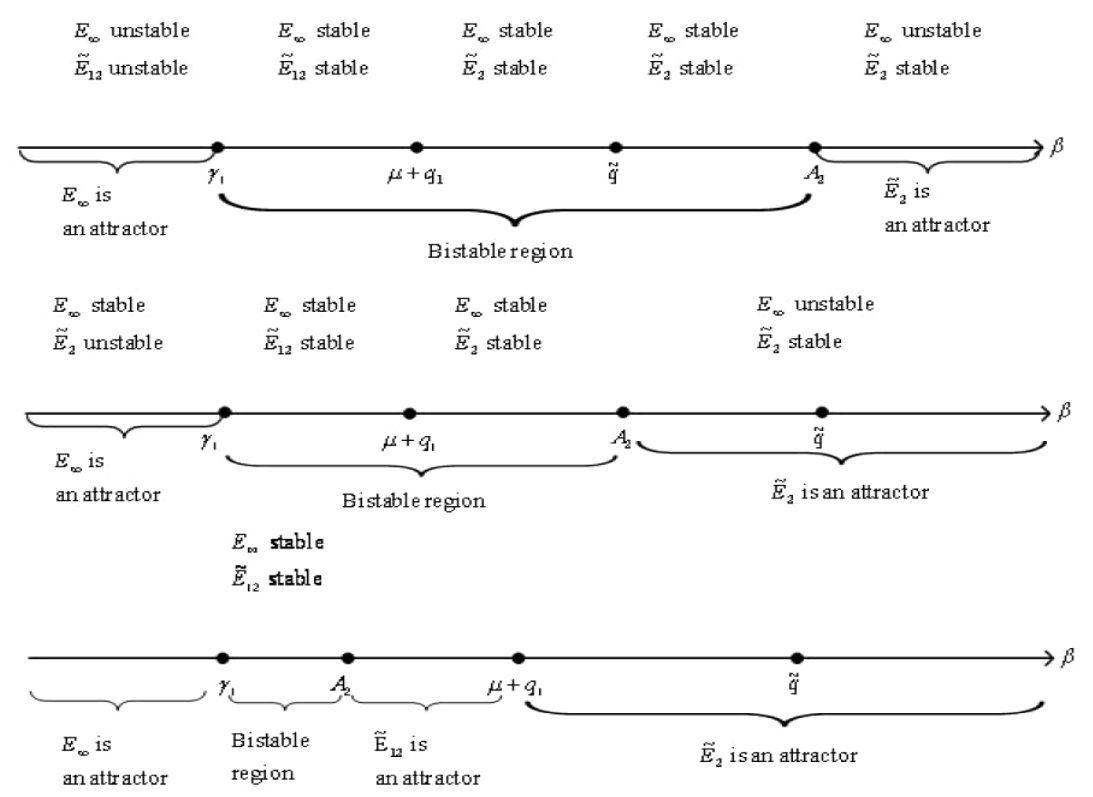

FIG. 4.4. Stability of $\widetilde{E_{12}}$ and $E_{\infty}$ when $\widetilde{q}>\mu+q_{1}>\gamma_{1}$.

Case 2. $\widetilde{q}<\mu+q_{1}, \gamma_{1}<\mu+q_{1}$, and we have two subcases:

Subcase (i). $\gamma_{1}<\widetilde{q}$. The stability of $\widetilde{E_{0}}, \widetilde{E_{12}}$, and $E_{\infty}$ is as follows.

- $\gamma_{1}<q<A_{2}<\mu+q_{1}$.

If $\beta<\gamma_{1}$, then $E_{\infty}$ is an attractor; if $\gamma_{1}<\beta<\widetilde{q}$, then $E_{\infty}, \widetilde{E}_{12}$ are stable; if $\widetilde{q}<\beta<A_{2}$, then $E_{\infty}, \widetilde{E}_{0}$ are stable; if $\beta>A_{2}$, then $\widetilde{E}_{0}$ is an attractor.

- $\gamma_{1}<\widetilde{q}<\mu+q_{1}<A_{2}$.

Same as above.

- $\gamma_{1}<A_{2}<\widetilde{q}<\mu+q_{1}$.

If $\beta<\gamma_{1}$, then $E_{\infty}$ is an attractor; if $\gamma_{1}<\beta<A_{2}$, then $\widetilde{E}_{12}, E_{\infty}$ are stable; if $A_{2}<\beta<\widetilde{q}$, then $\widetilde{E}_{12}$ is an attractor; if $\beta>\widetilde{q}$, then $\widetilde{E}_{0}$ is an attractor.

Subcase (ii). $\gamma_{1}>\widetilde{q}$. Then $A_{2}>\widetilde{q}$. If $\beta<\widetilde{q}$, then $E_{\infty}$ is an attractor. If $\widetilde{q}<\beta<A_{2}$, then $\widetilde{E}_{0}$ and $E_{\infty}$ are stable. If $\beta>A_{2}$, then $\widetilde{E}_{0}$ is an attractor. 
Case 3. $\widetilde{q}<\mu+q_{1}<\gamma_{1}$, and $\gamma_{1}>\widetilde{q}$. Then $A_{2}>\widetilde{q}$, and we have a result similar to that in Subcase (ii).

5. Concluding remarks. For the model without quarantine but with behavior change due to public response to the severity of the disease, we have shown that the local stability condition in Theorem 3.4 is equivalent to the condition that the basic reproduction number $R_{0}$ given in (2.15) with $\alpha_{P}=0$ is less than 1 . If $\beta c>\sigma_{1}+\rho_{1}+\gamma_{3}$, the epidemic would persist without public response; however, if the magnitude of public response, as measured by the parameter $a$, is sufficiently large so that $R_{0}<1$, the reduction of infections through public response will be large enough to drive the epidemic down to a disease-free state.

For the model with both quarantine and behavior change, the dynamics is much more complicated. The effective reproduction number with quarantine $R_{Q}$ in (2.16) gives local stability of DFE when $R_{Q}<1$. However, there are ranges of the parameters which would lead to bistable steady states, i.e., one locally stable DFE and another locally stable endemic equilibrium. In such cases, we conjecture that there is a saddle point with two-dimensional stable manifold. As an illustration, we give the following numerical example.

We let $\alpha_{A}=0.1, \widetilde{\beta}=0.5, \widetilde{q}=0.3, \gamma_{1}=0.4, q_{1}=0.2, \mu=0.2$, and use the initial values of $S(0)=1, E(0)=0, Q_{A}(0)=0, I(0)=1$, i.e., one infective case entering a totally susceptible population of one individual so that $\left(W_{1}(0), W_{2}(0), W_{3}(0)\right)=$ $(1,0,0)$. The result is given in Figure 5.1, where the system goes to the endemic equilibrium.

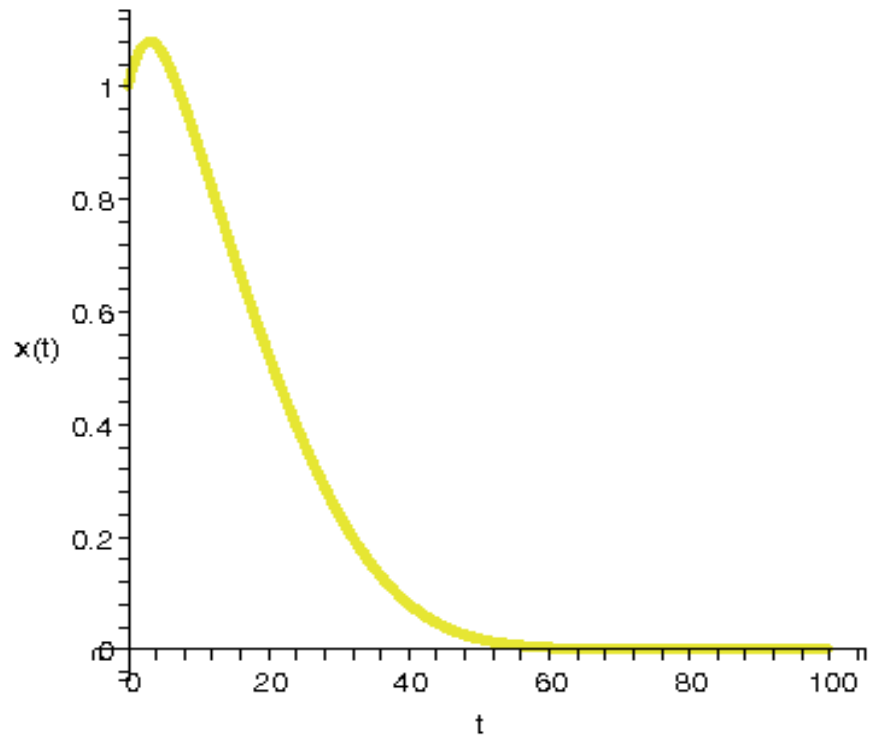

FIG. 5.1. Numerical example with $\alpha_{A}=0.1, \widetilde{\beta}=0.5, \widetilde{q}=0.3, \gamma_{1}=0.4, q_{1}=0.2, \mu=0.2$, and initial population $S(0)=1, E(0)=0, Q_{A}(0)=0, I(0)=1$, where system approaches endemic equilibrium. $X(t)$ is $W_{1}(t)=S(t) / I(t)$, which goes to zero.

However, if we let the initial values be $S(0)=10, E(0)=0, Q_{A}(0)=0, I(0)=1$, i.e., 10 infective cases entering a totally susceptible population of 100 individuals, so that $\left(W_{1}(0), W_{2}(0), W_{3}(0)\right)=(10,0,0)$, the system will approach DFE as shown in 
Figures 5.2 and 5.3. Note that for this set of parameters, $A_{2}=0.56>\widetilde{\beta}=0.5>$ $\widetilde{q}=0.3, \mu+q_{1}=0.2+0.2>\widetilde{q}=0.3, \gamma_{1}=0.4>\widetilde{q}=0.3$. Moreover, Case 3 of Theorem 4.8 holds for this data. Therefore we have bistability where both $E_{0}=(0,0,0)$ and $E_{\infty}$ are locally stable.

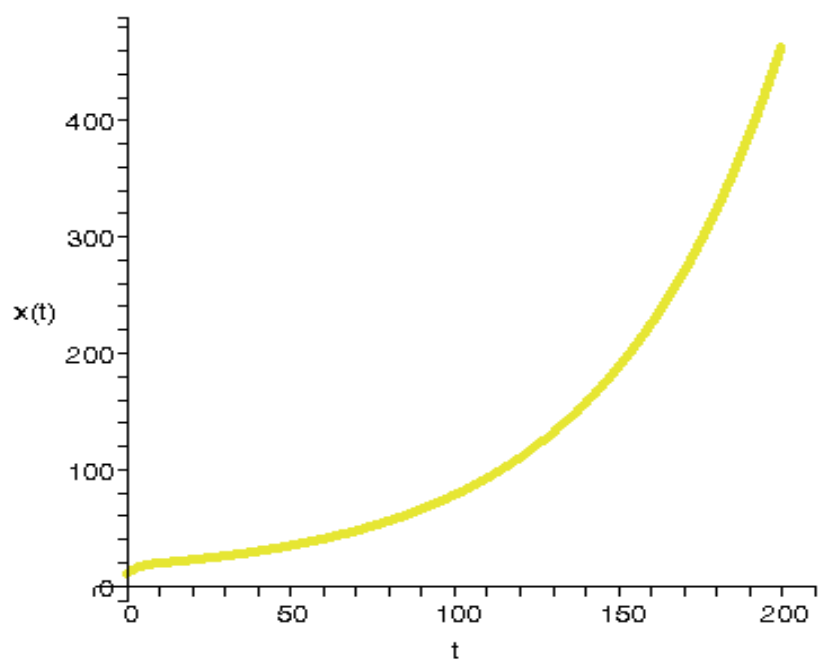

FIG. 5.2. Numerical example with $\alpha_{A}=0.1, \widetilde{\beta}=0.5, \widetilde{q}=0.3, \gamma_{1}=0.4, q_{1}=0.2, \mu=0.2$, and initial population $S(0)=10, E(0)=0, Q_{A}(0)=0, I(0)=1$, where the system approaches DFE. $X(t)$ is $W_{1}(t)=S(t) / I(t)$, which goes to a nonzero equilibrium, and $S(t) \rightarrow 14.58322$.

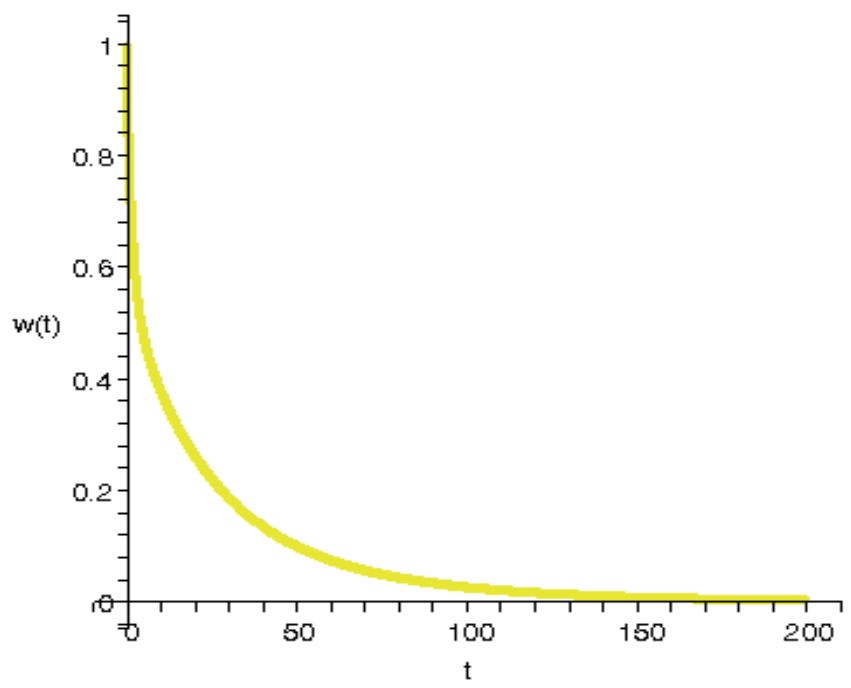

FIG. 5.3. Numerical example with $\alpha_{A}=0.1, \widetilde{\beta}=0.5, \widetilde{q}=0.3, \gamma_{1}=0.4, q_{1}=0.2, \mu=0.2$, and initial population $S(0)=10, E(0)=0, Q_{A}(0)=0, I(0)=1$, where the system approaches DFE. $W(t)$ is $I(t)$, which goes to zero.

The epidemiological interpretation is most interesting. In an epidemic where there is public response to the increasing severity of the epidemic to cut down infections 
through individual behavior change, sufficient decrease of the infection rate through the parameter $a$ would be enough to contain the epidemic, regardless of the initial population sizes at the onset of outbreak.

Suppose that, in addition to the severity-dependent public response, quarantine is implemented. If the adherence to quarantine to decrease contact rate $c$ and transmission probability $\beta$ is satisfactory, the effective infection rate $\widetilde{\beta}=\frac{\beta c}{1+a N}$ is sufficiently lowered. Case 1 in Theorem 4.8 will be satisfied along with $\widetilde{\beta}<\widetilde{q}$, and the epidemic can be successfully contained, again regardless of the initial population sizes at the onset of outbreak. If, on the other hand, $\widetilde{\beta}$ is not sufficiently lowered and we have Case 2 of Theorem 4.8, the epidemic will persist and the susceptible population $S(t)$ will be depleted eventually. There is the third scenario, where $\widetilde{\beta}$ is decreased but not sufficiently so, to less than $A_{2}$ but greater than $\widetilde{q}$. Then the system could approach either the DFE or the endemic steady state, depending on the initial values of the system.

In the worst case scenario, if the quarantine were not adhered to faithfully, the false sense of security brought about by the quarantine that all infective persons were in quarantine could lead to increased $\widetilde{\beta}$. Thus the quarantine might in fact have an adverse effect by contributing to the persistence of the epidemic. For illustration, we consider the hypothetical case where $\beta<\widetilde{q}=\sigma_{1}+\rho_{1}+\gamma_{3}$. Since $\beta>\widetilde{\beta}$ when $c=1$, from Theorem 3.4 we know the system goes to DFE with no quarantine implemented. However, for Case 1 (Figure 4.4), Subcase 3, when $\mu+q_{1}<\beta<\widetilde{q}$ the system will converge to the endemic equilibrium. This demonstrates the (distinct) possibility that, under appropriate parameter values, a quarantine program which is not sufficiently comprehensive $\left(q_{1}<\beta-\mu\right)$ could have the adverse effect of causing a system which would have approached DFE without quarantine to converge to the endemic equilibrium instead.

We should note, however, that $\widetilde{\beta}=\frac{\beta c}{1+a N}$, where $N$ is the constant total population size, which numbers in at least millions whether in Hong Kong, Singapore, Taipei, Beijing, or Toronto. In [27], it was determined that $a=0.0013$ and $c \beta=0.429$ for Taiwan's SARS outbreak. Subsequently the real value for $\widetilde{\beta}$ is of the order $0.429 /\left(1+0.0013 * 10^{6}\right) \ll 1$, making it most unlikely for either Case 2 (asymptotically stable endemic equilibrium) or Case 3 (bistability) in Theorem 4.8 to prevail. In other words, the modeling results indicate that for an infectious disease with infectivity and patterns of transmission typical of SARS, the outbreak can always be eradicated by implementing border control of imported cases and limited quarantine, along with the public's social response to avoid infections.

It is also interesting to note that if $\alpha_{A}=0$, the stability condition for $E_{\infty}$ becomes $\beta<\widetilde{q} \mu /\left(\mu+q_{1}\right)$. Hence quarantine is always beneficial and an effective Level $\mathrm{A}$ quarantine is always helpful in containing the epidemic. However, if for some disease unlike SARS in its ability to infect during the asymptomatic stage, some fraction of the quarantined population is not fully isolated and can still infect others (i.e., $\alpha_{A}>0$ ), then quarantine might also affect the outbreak adversely. A numerical example of this scenario is as follows: Let $\alpha_{A}=0.1, \beta=0.5, \mu=0.5, \widetilde{q}=0.7$, $q_{1}=0.1, \gamma_{1}=0.01, A_{2}=0.35$. Here $\widetilde{E}_{12}=(0.08,0.4,+\infty)$ is a global attractor as in Case 1, Subcase 3 in Figure 4.4. However, if there is no quarantine (i.e., $q_{1}=0$ and hence $\alpha_{A}=0$ ), DFE is the global attractor. Additional examples of this type can be observed in Case 1, Subcases 2 and 3 (Figure 4.4), where $\widetilde{E}_{2}$ can become the global attractor for the appropriate parameter range of $\beta$, as well as in Case 2, Subcase (i), where again it is possible for $\widetilde{E}_{12}$ to become a global attractor. Note that 
a condition for these cases to emerge is $\widetilde{q}>A_{2}$ or, equivalently, $\gamma_{1} / \widetilde{q}<\alpha_{A}$. Hence if there is a nonzero reduction in the infection rate of the quarantined class $\alpha_{A}$ which is larger than the ratio of the progression rate of the quarantined persons $\gamma_{1}$ to the removal rate of the unquarantined infectives $\widetilde{q}$, an adverse effect could take place with implementation of quarantine. To keep this possibility from occurring, one would need either (i) significant reduction of infection by the quarantined individuals (small $\alpha_{A}$ ) or (ii) quick isolation of quarantined persons at onset (large $\gamma_{1}$ ) compared to the removal of the infective class (small $\widetilde{q}$ ). Similar possible adverse effects of intervention measures have also been observed in other theoretical models of infectious diseases (e.g., [30, 31]).

Going back to the quarantine for SARS, we assume that $\alpha_{P}=\alpha_{A}=0$. If all other pertinent parameters remain the same, we have $R_{Q}=R_{0} \mu /\left(\mu+q_{1}\right)$ from (2.15)-(2.16). That is, the implementation of quarantine would give the mean reproduction number of an infective individual a factor of $\mu /\left(\mu+q_{1}\right)$, where $q_{1}$ is the effective quarantine rate. That is, through quarantine alone, the mean reproduction number of an infective individual is reduced by a factor of $1-\mu /\left(\mu+q_{1}\right)$.

In a data-based modeling study where the pertinent parameters were estimated from the Taiwan SARS data [27], the quarantine rate $q_{1}$ was estimated to be 0.0277 . It was reported by Donnelly et al. [32] that the maximum likelihood estimate for the mean time from exposure to onset of symptoms is 6.37 days. Hence the mean progression rate from exposure to onset is approximately $\mu=1 / 6.37=0.157$. Making use of the two estimates, we conclude that if all other parameters remain unchanged, the quarantine in Taiwan would result in a reduction of $15 \%\left(\mu /\left(\mu+q_{1}\right)=0.850\right)$ in the mean reproduction number by an infective individual. Given that current studies of SARS indicate that the basic reproduction numbers $R_{0}$ in all the SARS-affected areas were greater than 2 at the beginning of the outbreak in 2003, one can conclude that quarantine alone would not have been able to contain the epidemic (i.e., reduce $R_{0}$ to less than 1) in Taiwan. For a given affected area with a basic reproduction number $R_{0}$, we need to have an effective quarantine rate of $q_{1}>q_{1}^{*}=0.157\left(R_{0}-1\right)$ for $R_{Q}$ to be less than 1 . Using the estimated values of $R_{0}$ for Hong Kong, Toronto, and Taiwan in current literature, we give in Table 5.1 the effective quarantine rate $q_{1}^{*}$ needed in the affected areas to reduce the reproduction number to less than 1 , if all else remains the same. Note that the estimate for Taiwan [13] assumes that a symptomatic patient is infective from onset to classification as a probable case followed by isolation. If we assume the patient is not infective during the first two days of onset as suggested by some studies (see [2]), the reproduction number is reduced to 3.56 and subsequently $q_{1}^{*}=0.402$.

TABLE 5.1

\begin{tabular}{|l|c|c|}
\hline Affected area & $\begin{array}{c}\text { Reproduction number } \\
\text { [literature cited] }\end{array}$ & $\begin{array}{c}\text { Effective quarantine rate } q_{1}^{*} \\
\text { needed to contain outbreak }\end{array}$ \\
\hline Hong Kong & $2.7[32]$ & 0.267 \\
& $3[10]$ & 0.314 \\
\hline Toronto & $3.3[33]$ & 0.361 \\
\hline Taiwan & $4.23[13]$ & 0.507 \\
\hline
\end{tabular}

Since the SARS-CoV virus does not appear to be infective before onset of symptoms [2], quarantine does not directly prevent infections by the exposed individuals during the quarantine period. However, studies on SARS quarantine data in Taiwan [8] indicate that quarantined persons are significantly more quickly diagnosed 
and hospitalized as compared to the unquarantined individuals. Hence the effectiveness of quarantine for infectious diseases like SARS, for which no infection is being prevented during the quarantine period, can only be indirect and therefore must be combined with other intervention measures in order to fully contain the outbreaks.

Acknowledgments. The authors are grateful for constructive discussions with Roy Anderson, John Glasser, Chwan-Chuan King, and Fred Brauer which helped formulate some of the ideas for this work. Ying-Hen Hsieh would like to thank MITACS (Canada) for their generous financial support while attending the MITACS SARS meetings in Banff, Canada, where several of the abovementioned discussions took place.

\section{REFERENCES}

[1] World Health Organization, Summary of Probable SARS Cases with Onset of Illness from 1 November 2002 to 31 July 2003, http://www.who.int/csr/sars/country/table2003_09_23/ en/ (26 September 2003).

[2] World Health Organization, Consensus Document on the Epidemiology of Severe Acute Respiratory Syndrome (SARS), http://www.who.int/csr/sars/en/WHOconsensus.pdf (17 October 2003).

[3] M. EnSERINK, SARS: A pandemic prevented, Science, 302 (2003), p. 2045.

[4] J. OU, Q. LI, AND G. ZENG, Efficiency of quarantine during an epidemic of severe acute respiratory syndrome-Beijing, China, 2003, MMWR, 52 (2003), pp. 1037-1040.

[5] X. PAng, Z. Zhu, F. Xu, J. GuO, X. Gong, ET AL., Evaluation of control measures implemented in the severe acute respiratory syndrome outbreak in Beijing, 2003, JAMA, 290 (2003), pp. 3215-3221.

[6] B. DiAmOND, SARS spreads new outlook on quarantine models, Nat. Med., 9 (2003), p. 1441.

[7] M. L. Lee, C. J. Chen, I. J. Su, K. T. Chen, C. C. Yeh, C. C. King, et Al., Use of quarantine to prevent transmission of severe acute respiratory syndrome-Taiwan 2003, MMWR, 52 (2003), pp. 680-683.

[8] Y. H. Hsieh, C. C. King, M. S. Ho, C. W. S. Chen, J. Y. Lee, F. C. Liu, Y. C. Wu, and J. S. J. Wu, Quarantine for SARS, Taiwan, Emerg. Infect. Dis., 11 (2005), pp. 278-282.

[9] L. O. Gostin, R. BAyer, AND A. L. FAIrChild, Ethical and legal challenges posed by severe acute respiratory syndrome: Implications for the control of severe infectious disease threats, JMAM, 290 (2003), pp. 3229-3237.

[10] M. Lipsitch Et AL., Transmission dynamics and control of severe acute respiratory syndrome, Science, 300 (2003), pp. 1966-1970.

[11] S. RILEY ET AL., Transmission dynamics of the etiological agent of SARS in Hong Kong: Impact of public health interventions, Science, 300 (2003), pp. 1961-1966.

[12] J. O. Lloyd-Smith, A. P. Galvani, And W. M. Getz, Curtailing transmission of severe acute respiratory syndrome within a community and its hospital, Proc. R. Soc. Lond. B Biol. Sci., 270 (2003), pp. 1979-1989.

[13] Y. H. Hsien, C. W. S. Chen, And S. B. Hsu, SARS outbreak, Taiwan 2003, Emerg. Infect. Dis., 10 (2004), pp. 201-206.

[14] Y. H. Hsieh, J. Y. LeE, AND H. L. Chang, On SARS epidemiology, cumulative case curve, and logistic-type model: Ascertaining effectiveness of intervention and predicting case number, Emerg. Infect. Dis., 10 (2004), pp. 1165-1167.

[15] C. A. Donnelly, M. C. Fisher, C. Fraser, A. C. Ghani, S. Riley, N. M. Ferguson, AND R. M. Anderson, Epidemiological and genetic analysis of severe acute respiratory syndrome, Lancet Infect. Dis., 4 (2004), pp. 672-683.

[16] E. H. Kaplan, D. L. Craft, And L. M. Wein, Analyzing bioterror response logistics: The case of smallpox, Math. Biosci., 185 (2003), pp. 33-72.

[17] L. Sattenspiel And D. A. Herring, Simulating the effect of quarantine on the spread of the 1918-19 flu in central Canada, Bull. Math. Biol., 65 (2003), pp. 1-26.

[18] H. Hethcote, Z. Ma, and S. LiaO, Effects of quarantine in six endemic models for infectious diseases, Math. Biosci., 180 (2002), pp. 141-160.

[19] E. H. Kaplan, D. L. CRAFt, And L. M. Wein, Emergency response to a smallpox attack: The case for mass vaccination, Proc. Natl. Acad. Sci. USA, 99 (2002), pp. 10935-10940.

[20] M. I. Meltzer, I. Damon, J. W. LeDuc, and J. D. Millar, Modeling potential responses to smallpox as a bioterrorist weapon, Emerg. Infect. Dis., 7 (2001), pp. 959-969. 
[21] M. J. Keeling and C. A. Gilligan, Metapopulation dynamics of bubonic plague, Nature, 407 (2000), pp. 903-906.

[22] P. Rohani, C. J. Green, N. B. Mantilla-Beniers, and B. T. Grenfell, Ecological interference between fatal diseases, Nature, 422 (2003), pp. 885-888.

[23] D. Clancy, Optimal intervention for epidemic models with general infection and removal rate functions, J. Math. Biol., 39 (1999), pp. 309-331.

[24] C. S. Holling, The functional response of predators to prey density and its role in mimicry and population regulation, Mem. Entolmol. Soc. Can., 45 (1965), pp. 1-60.

[25] F. Brauer and P. VAn Den Driessche, Models for transmssion of disease with immigration of infectives, Math. Biosci., 171 (2001), pp. 143-154.

[26] P. van den Driessche and J. Watmough, Reproduction numbers and sub-threshold endemic equilibria for compartmental models of disease transmission, Math. Biosci., 180 (2002), pp. 29-48.

[27] Y. H. Hsien, C. C. King, C. W. S. Chen, M. S. Ho, S. B. Hsu, and Y. C. Wu, On the Impact of Intervention Measures and Public Response for Severe Acute Respiratory Syndrome Outbreak, preprint, National Chung Hsing University, Taichung, Taiwan.

[28] M. S. Ho AND I. J. Su, Preparing to prevent severe acute respiratory syndrome and other respiratory infections, Lancet Infect. Dis., 4 (2004), pp. 684-689.

[29] W. A. Coppell, Stability and Asymptotic Behavior of Solutions of Differential Equations, Heath, Boston, 1965.

[30] R. M. Anderson, S. Gupta, and R. M. May, Potential of community-wide chemotherapy or immunotherapy to control the spread of HIV-1, Nature, 350 (1991), pp. 356-359.

[31] Y. H. Hsieh and J. Velaso-Hernanadez, Community treatment of HIV-1: Initial and asymptotic dynamics, BioSystems, 35 (1995), pp. 75-81.

[32] C. A. Donnelly, A. C. Ghani, G. M. Leung, A. J. Hedley, C. Fraser, S. Riley, L. J. Abu-Raddad, L. M. Ho, T. Q. Thach, P. Chau, K. P. Chan, T. H. Lam, L. Y. Tse, T. Tsang, S. H. Liu, J. H. Kong, E. M. Lau, N. M. Ferguson, and R. M. Anderson, Epidemiological determinants of spread of causal agent of severe acute respiratory syndrome in Hong Kong, Lancet, 361 (2003), pp. 1761-1766.

[33] J. Wallinga, Presentation at the Global Meeting on the Epidemiology of SARS, World Health Organization, Geneva, Switzerland, 2003. 